\title{
Alcohol consumption and risk of cardiovascular disease, cancer and mortality: a prospective cohort study
}

Xinyuan Zhang ${ }^{1}$, Yan Liư ${ }^{2}$, Shanshan $\mathrm{Li}^{3}$, Alice H. Lichtenstein ${ }^{4}$, Shuohua Chen², Muzi Na', Susan Veldheer ${ }^{5}$, Aijun Xing ${ }^{2}$, Yanxiu Wang ${ }^{2}$, Shouling $\mathrm{Wu}^{2^{*}}$ and Xiang Gao ${ }^{1^{*}}$ (D)

\begin{abstract}
Background: Studies regarding whether light to moderate alcohol consumption is associated with a lower risk of cardiovascular diseases (CVD) have generated mixed results. Further, few studies have examined the potential impact of alcohol consumption on diverse disease outcomes simultaneously. We aimed to prospectively study the dose-response association between alcohol consumption and risk of CVD, cancer, and mortality.

Methods: This study included 83,732 adult Chinese participants, free of CVD and cancer at baseline. Participants were categorized into 6 groups based on self-report alcohol consumption: 0, 1-25, 26-150, 151-350, 351-750, and $>750 \mathrm{~g}$ alcohol/wk. Incident cases of CVD, cancers, and mortality were confirmed by medical records. Hazard ratios (HRs) for the composite risk of these three outcomes, and each individual outcome, were calculated using Cox proportional hazard model.

Results: During a median follow-up of 10.0 years, there were 6411 incident cases of CVD, 2947 cancers and 6646 deaths. We observed a J-shaped relation between alcohol intake and risk of CVD, cancer, and mortality, with the lowest risk at $25 \mathrm{~g} / \mathrm{wk}$., which is equivalent to $\sim 2$ servings/wk. Compared to consuming $1-25 \mathrm{~g} / \mathrm{wk}$., the adjusted HR for composite outcomes was 1.38 (95\% confidence interval (Cl):1.29-1.49) for non-drinker, 1.15 (95\% Cl: 1.041.27) for 26-150 g/wk., 1.22 (95\% Cl: 1.10-1.34) for 151-350 g/wk., 1.33 (95\% Cl: 1.21-1.46) for 351-750 g/wk., and 1.57 (95\% Cl: 1.30-1.90) for $>750 \mathrm{~g} / \mathrm{wk}$. after adjusting for age, sex, lifestyle, social economic status, and medication use.
\end{abstract}

Conclusions: Light alcohol consumption at $\sim 25 \mathrm{~g} / \mathrm{wk}$ was associated with lower risk of CVD, cancer, and mortality than none or higher consumption in Chinese adults.

Keywords: Alcohol consumption, Cardiovascular disease, Cancer, Mortality, Nutritional epidemiology

\section{Introduction}

Worldwide in 2016, current drinkers aged 15 years and over consumed an average of $230 \mathrm{~g}$ of alcohol per week [1]. Although the prevalence of alcohol intake worldwide

\footnotetext{
* Correspondence: drwusl@163.com; xxg14@psu.edu

²Department of Cardiology, Kailuan General Hospital, 57 Xinhua East Rd, Tangshan 063000, China

${ }^{1}$ Department of Nutritional Sciences, The Pennsylvania State University, 109 Chandlee Lab, University Park, PA 16802, USA

Full list of author information is available at the end of the article
}

has decreased since 2000, in contrast, China has experienced a decrease in the prevalence of lifetime abstainers and an increase in total alcohol per capita consumption [1].

The association between alcohol consumption and chronic health outcomes is controversial. Early studies have consistently reported that low to moderate alcohol consumption (i.e., $\leq \sim 200 \mathrm{~g} / \mathrm{wk}$ ) is associated with lower risk of cardiovascular disease (CVD) than those who

C C The Author(s). 2021 Open Access This article is licensed under a Creative Commons Attribution 4.0 International License, which permits use, sharing, adaptation, distribution and reproduction in any medium or format, as long as you give appropriate credit to the original author(s) and the source, provide a link to the Creative Commons licence, and indicate if changes were made. The images or other third party material in this article are included in the article's Creative Commons licence, unless indicated otherwise in a credit line to the material. If material is not included in the article's Creative Commons licence and your intended use is not permitted by statutory regulation or exceeds the permitted use, you will need to obtain permission directly from the copyright holder. To view a copy of this licence, visit http://creativecommons.org/licenses/by/4.0/ The Creative Commons Public Domain Dedication waiver (http://creativecommons.org/publicdomain/zero/1.0/) applies to the data made available in this article, unless otherwise stated in a credit line to the data. 
consume no alcohol and or have very high consumption [2-4]. Recently, however, cumulative evidence from combined perspective studies [5] and genetic epidemiological studies $[6,7]$ have challenged this traditional Jshape relationship, reporting a linear positive association between alcohol intake and CVD risk.

Heavy intake of alcohol has been causally linked to cancers of the oral cavity, pharynx, esophagus, liver, larynx, colorectum, female breast, and possibly to cancers of the pancreas and prostate [8]. In contrast, some observational studies have suggested that light to moderate alcohol is associated with lower risk for total cancer and cancers of lung, cervix, thyroid, and kidney [9].

Although many studies have reported a positive association between alcohol intake and fatalities from accidents, intoxication, indulgence or self-harm, and altered motor function [10], some studies have reported that consumption of up to $100 \mathrm{~g} / \mathrm{wk}$. of alcohol was inversely associated with all-cause mortality [11]. Currently there are few available reports on the relationship between alcohol intake and site-specific cancer mortality, with little evidence of a beneficial effect related to low to moderate alcohol intake [11]. This gap makes it difficult to draw conclusions on the relationship between alcohol intake and overall health outcome.

Alcohol consumption, and its impact on chronic diseases and mortality, is heavily influenced by genes, lifestyle, and socio-economic status (SES) [2]. Aldehyde dehydrogenase (ALDH2) deficiency, the lack of a major enzyme responsible for in vivo alcohol oxidation, is common among Asian populations, while being rare in Western populations [12]. Meanwhile, Asian countries rank high in several alcohol-attributed diseases, included cancers of liver and esophagus [13]. The high level of disease risk and genetic predisposition suggests that specific attention to this population is warranted. To date, most cohort studies have been conducted in Western and high-income countries $[2-5,11,14,15]$, leaving Asian and middle- to low-income countries underrepresented. Further, previous meta-analyses and pooledanalyses included populations with vast variability in SES $[5,9,16-18]$. Inclusion of results from a variety of populations and study designs inevitably introduces heterogeneity to the results.

We aim to assess the relationship between alcohol consumption and adverse health outcomes, including cardiovascular diseases, cancers (total and site-specific), and mortality, in a homogenous population. We used a composite of CVD, cancer and total mortality as primary outcomes, to illustrate an overall pattern of alcohol consumption and chronic disease risk, as did previously [19, 20]. As a secondary analysis, associations between alcohol intake and each condition were examined. Our hypothesis was that low to moderate alcohol consumption would be associated with lower risk of major chronic diseases and mortality, compared to none or high alcohol consumption.

\section{Methods \\ Study population}

The Kailuan Study is an ongoing prospective cohort based on the Kailuan community in the city of Tangshan, northeast China (trial registration number: ChiCTR-TNRC-11001489) [21]. In brief, 101,510 participants (81,110 men and 20,400 women, 18 years or older) were recruited into the study from June 2006 to October 2007 and were followed-up biennially. At baseline and every follow-up, participants completed a clinical examination, including physical and laboratory measurements, and self-administrated questionnaires. In the current study, we included participants from the 2006-2007 baseline and their follow-up information until 20162017.

Exclusion criteria were 1) not answering questions regarding alcohol consumption, or 2) having history of CVD or cancer at baseline. Out of the 101,510 participants, 14,316 had missing response to questions regarding alcohol consumption at baseline (2006-2007). A baseline comparison between these 14,316 excluded participants and included participants was presented in Supplemental Table 1. We further excluded those who had had cardiovascular disease or cancer $(n=3845)$ prior to baseline. Included in the analyses were 83,732 participants (Supplemental Figure 1).

\section{Assessment of alcohol consumption}

A self-administered questionnaire were used to collect information on alcohol consumption [22]. Questions included 1) whether they consumed alcoholic beverages in the past 12 months, 2) if so, the beverage types (beer, wine or hard liquor), and 3) the amount and frequency of intake for each beverage type. Gram (g) of alcohol consumed per week was calculated by multiplying the average frequency (times per week) by the amount usually consumed for each beverage and its average alcohol content (assumed to be $5.0 \mathrm{~g}$ for $100 \mathrm{~g}$ beer, $12.0 \mathrm{~g}$ for $100 \mathrm{~g}$ wine, and $40.0 \mathrm{~g}$ for $100 \mathrm{~g}$ hard liquor). Gram of alcohol was categorized into 5 groups: 1-25, 26-150, 151-350, 351-750, and $>750 \mathrm{~g} / \mathrm{wk}$., based on previous published cut-off points [5]. Not drinking any alcoholic beverages in the past 12 months were categorized as $0 \mathrm{~g}$ / wk.

Our previous analysis showed a dose-response relationship between alcohol consumption and high-density lipoprotein cholesterol (HDL-C) concentrations in the Kailuan Study participants [22], which further support the validity of the self-reported alcohol consumption data collected as part of the Kailuan study. 


\section{Assessment of outcomes}

The primary end point was a composite of CVD, cancer, and mortality, whichever happened first $[19,20]$. Secondary outcomes were individual diseases/mortality, including myocardial infarction, stroke, heart failure and atrial fibrillation; alcohol-related cancer (including oral cavity and pharynx, larynx, esophageal, colorectum, anal, salivary gland, liver, and female breast), and other cancers (referred to as "non-alcohol-related cancers") [9, 23]; and CVD-attributed, cancer-attributed and othercause mortality.

Incident cases of CVD, cancer, and death were confirmed by review of insurance and medical records, as detailed elsewhere [21, 24, 25]. Questions related to history of CVD and cancer were included in the biennial questionnaire. All participants were linked to the Municipal Social Insurance Institution and the Hospital Discharge Register for incidence of CVD and cancer, which covers all the Kailuan study participants. Medical records from 2006 to 2016 of all patients from the 11 registered hospitals of Kailuan Study were reviewed by study physicians, including 3 cardiologists for CVD ascertainment and 3 oncologists for cancer ascertainment. Heart failure was diagnosed according to Framingham criteria [26]. Atrial fibrillation was diagnosed using a standard 12-lead electrocardiogram [27]. Cancer was diagnosed using pathological and imaging diagnosis and coded by International Classification of Diseases, 10th version (ICD-10) [28]. Mortality information was collected from provincial vital statistics offices. Blinded clinicians reviewed death certificates and coded cause of death according to ICD-10 codes [29].

\section{Assessment of covariates}

We used questionnaires data for information on SES (eg, marital status, education level, occupation type, and household income per capita), medical history, use of medications (eg, anti-hypertensive, anti-diabetes and lipid-lowering agents), and lifestyle factors (eg, smoking status, physical exercise, and salt intake), as detailed elsewhere [21, 28, 29]. Marital status was categorized into single, first marriage, and other; education level was categorized into elementary school or below, middle or high school, or college or above; household income per capita was categorized into $\leq 500,501-1000$, or $>1000$ Chinese yuan/month; smoking status was categorized into never, past, and current smoker; physical activity was categorized into $<1,1-3$, or $\geq 4$ times/week; sodium intake was categorized into $<6,6-9.9$, or $\geq 10 \mathrm{~g} /$ day.

Clinical examinations were performed at $7-9$ am by trained nurses. Anthropometry examinations (eg, height and weight) were conducted. Body mass index (BMI) was calculated as weight $(\mathrm{kg}) /$ height $^{2}\left(\mathrm{~m}^{2}\right)$. Systolic and diastolic blood pressure were measured on standard desktop mercury sphygmomanometers. Overnight fasting $(>8 \mathrm{~h})$ blood samples were collected to measure serum concentrations of fasting blood glucose (FBG) with the hexokinase/glucose-6-phosphate dehydrogenase method, HDL-C and low- density lipoprotein cholesterol (LDL-C) with direct test assay (Mind Bioengineering Co. Ltd., Shanghai, China), uric acid with commercial kit (Ke Hua Biological Engineering Corporation, Shanghai, China), and high sensitive C-reactive protein (hs-CRP) with high sensitivity nephelometry assay (Cias Latex CRP-H; Kanto Chemical, Tokyo, Japan), which were analyzed by auto-analyzer (Hitachi 747; Hitachi, Tokyo, Japan) at the central laboratory of Kailuan hospital [24, 30]. Concentration of hs-CRP was log-transformed for statistical analysis due to skewed distribution.

\section{Statistical analysis}

Baseline characteristics were compared using one-way Analysis of Variance for continuous variables and chisquare tests for categorical variables.

We computed the person-year of follow-up for each participant that contributed to each outcome from 2006 baseline to the date of diagnosis or the end of follow-up (December 31, 2016), whichever came first.

Hazard ratios (HR) and 95\% confidence intervals (CI) for each alcohol consumption category were calculated using a Cox proportional-hazards model, adjusting for age, sex, SES, lifestyle factors, medication history, BMI and FBG concentration. The group $1-25 \mathrm{~g} / \mathrm{wk}$. of alcohol consumption was used as reference group. Trends across groups were assessed in models using the median alcohol consumption in each group as a continuous variable. To reduce the possibility of reverse-causality, we further conducted a 2-year lag analysis by excluding incident cases that occurred within the first 2 years of follow-up. The multiplicative interactions were tested between alcohol consumption and age, sex, smoking status, and occupation. Because very low alcohol intake is unlikely to have a biological effect, and the apparent benefit in this group could be due to confounding, we also conducted a sensitivity analysis by excluding participants with 1-4 g alcohol/wk. (i.e., 1 drink/mo).

We further constructed a restricted cubic spline model to display the dose-response association with 95\% CIs between continuous alcohol consumption and major chronic disease. Data were analyzed using SAS version 9.4 (SAS Institute Inc., US).

\section{Results}

Of the total 83,732 participants who were included in the analyses, the mean age was $51.3 \pm 12.3$ years, and 65 , $081(77.7 \%)$ were men. Baseline characteristics across the alcohol consumption groups are shown in Table 1. 
Table 1 Baseline characteristic comparison of 83,732 participants according to alcohol consumption

\begin{tabular}{|c|c|c|c|c|c|c|}
\hline \multirow[t]{2}{*}{ Alcohol consumption, g/wk } & 0 & $1-25$ & 26-150 & $151-350$ & $351-750$ & $>750$ \\
\hline & $n=58,706$ & $n=9258$ & $n=5024$ & $n=4520$ & $n=5522$ & $n=702$ \\
\hline Age, y & $52.12 \pm 12.3$ & $46.4 \pm 13.3$ & $48.5 \pm 12.3$ & $54.5 \pm 12.3$ & $50.4 \pm 9.1$ & $50.5 \pm 8.7$ \\
\hline Men, \% & 69.3 & 94.4 & 98.6 & 99.7 & 99.9 & 100.0 \\
\hline \multicolumn{7}{|l|}{ Socio-economic status } \\
\hline \multicolumn{7}{|l|}{ Marriage, \% } \\
\hline Single & 1.2 & 5.3 & 1.9 & 0.3 & 0.3 & 0.3 \\
\hline First marriage & 95.2 & 90.9 & 93.8 & 95.2 & 95.1 & 94.7 \\
\hline Other & 3.6 & 3.8 & 4.3 & 4.5 & 4.6 & 5.0 \\
\hline \multicolumn{7}{|l|}{ Education, \% } \\
\hline Elementary school or below & 9.2 & 8.9 & 11.7 & 18.5 & 14.3 & 17.2 \\
\hline Middle or high school & 86.0 & 73.5 & 73.2 & 78.3 & 82.1 & 79.1 \\
\hline College or above & 4.8 & 17.6 & 15.1 & 3.2 & 3.6 & 3.7 \\
\hline \multicolumn{7}{|l|}{ Occupation type, \% } \\
\hline Coal miner & 20.4 & 48.7 & 48.3 & 53.6 & 59.2 & 53.2 \\
\hline Blue-collar worker & 73.9 & 40.3 & 41.0 & 41.9 & 37.2 & 43.4 \\
\hline White-collar worker & 5.7 & 11.0 & 10.7 & 4.5 & 3.6 & 3.4 \\
\hline \multicolumn{7}{|l|}{ Household income per capita, \% } \\
\hline$<500 \mathrm{CNY} /$ month & 21.8 & 40.1 & 37.1 & 43.3 & 45.7 & 47.7 \\
\hline 501-1000 CNY /month & 73.5 & 47.9 & 50.0 & 50.2 & 46.6 & 42.3 \\
\hline > $1000 \mathrm{CNY} /$ month & 4.7 & 12.0 & 12.9 & 6.5 & 7.7 & 10.0 \\
\hline
\end{tabular}

Lifestyle

Beverage types, \%

Wine

Beer

Hard liquor

Smoking status, \%

$$
\text { Never }
$$

Past

Current

Physical exercise, \%

$$
<1 \text { times/week }
$$

1-3 times/week

$\geq 4$ times/week

Sodium intake, \%

$$
\begin{aligned}
& <6 \mathrm{~g} / \text { day } \\
& 6-9.9 \mathrm{~g} / \text { day }
\end{aligned}
$$$$
\geq 10 \text { /day }
$$

Medication

$\begin{array}{ll}\text { Anti-hypertension drugs } & 9.3 \\ \text { Anti-diabetes drugs } & 2.4 \\ \text { Lipid-lowering drugs } & 0.7\end{array}$

Clinical measurements

Body mass index, $\mathrm{kg} / \mathrm{m}^{2}$

Systolic blood pressure, $\mathrm{mmHg}$

$$
82.0
$$$$
3.7
$$

$25.0 \pm 3.6$

$131 \pm 21$
0.4

44.2

30.9

9.6

59.5

14.0

67.0

19.0

13.9

70.0

16.1

11.2

2.2

1.3

$25.0 \pm 3.4$

$126 \pm 19$
0.07

17.4

82.2

20.4

8.9

70.7

15.9

65.0

19.1

12.9

69.1

18.0

13.1

2.3

1.3

$25.5 \pm 3.5$

$129 \pm 20$

$$
0.01
$$$$
1.3
$$$$
99.8
$$

16.6

7.0

76.4

14.9

56.2

28.9

13.0

66.3

20.7

13.8

1.9

0.7

$24.8 \pm 3.2$

$136 \pm 21$

$25.1 \pm 3.2$

$135 \pm 20$

$25.2 \pm 3.3$

$134 \pm 21$ 
Table 1 Baseline characteristic comparison of 83,732 participants according to alcohol consumption (Continued)

\begin{tabular}{lllllll}
\hline Alcohol consumption, $\mathbf{g} / \mathbf{w k}$ & $\mathbf{0}$ & $\mathbf{1 - 2 5}$ & $\mathbf{2 6 - 1 5 0}$ & $\mathbf{1 5 1 - 3 5 0}$ & $\mathbf{3 5 1 - 7 5 0}$ & $>\mathbf{7 5 0}$ \\
& $\mathbf{n = 5 8 , 7 0 6}$ & $\mathbf{n = 9 2 5 8}$ & $\mathbf{n = 5 0 2 4}$ & $\mathbf{n = 4 5 2 0}$ & $\mathbf{n = 5 5 2 2}$ & $\boldsymbol{n}=\mathbf{7 0 2}$ \\
\hline Diastolic blood pressure, $\mathrm{mmHg}$ & $83 \pm 12$ & $82 \pm 11$ & $83 \pm 12$ & $86 \pm 12$ & $87 \pm 12$ & $86 \pm 12$ \\
Fasting blood glucose, $\mathrm{mmol} / \mathrm{L}$ & $5.48 \pm 1.75$ & $5.39 \pm 1.47$ & $5.52 \pm 1.63$ & $5.50 \pm 1.48$ & $5.56 \pm 1.52$ & $5.61 \pm 1.57$ \\
HDL-C, $\mathrm{mmol} / \mathrm{L}$ & $1.56 \pm 0.40$ & $1.47 \pm 0.37$ & $1.51 \pm 0.38$ & $1.59 \pm 0.41$ & $1.62 \pm 0.41$ & $1.58 \pm 0.40$ \\
LDL-C, $\mathrm{mmol} / \mathrm{L}$ & $2.28 \pm 0.89$ & $2.48 \pm 0.79$ & $2.52 \pm 0.86$ & $2.51 \pm 0.90$ & $2.56 \pm 0.90$ & $2.59 \pm 0.91$ \\
Uric acid, mmol/L & $275 \pm 79$ & $307 \pm 82$ & $318 \pm 86$ & $316 \pm 87$ & $318 \pm 88$ & $324 \pm 86$ \\
hs-CRP, $\mathrm{mg} / \mathrm{L}$ & $0.80(0.30,2.20)$ & $0.71(0.30,1.72)$ & $0.80(0.30,1.90)$ & $0.79(0.31,1.90)$ & $0.72(0.30,1.80)$ & $0.89(0.39,2.01)$
\end{tabular}

Values are mean \pm standard deviation, median with interquartile range, or frequency (percent)

$C N Y$ Chinese yuan, SBP systolic blood pressure, DBP diastolic blood pressure, $F B G$ fasting blood glucose, $H D L-C$ high-density lipoprotein cholesterol, $L D L-C$ lowdensity lipoprotein cholesterol, $h s$-CRP high-sensitivity C-reactive protein

\section{Alcohol and CVD, cancer, and mortality}

During a mean 10.0 year of follow-up period (interquartile range 9.7 to 10.2 years), there were 6411 CVD cases, 2947 cancer cases and 6646 deaths. Incident case of developing any composite major chronic diseases (CVD and cancer) or mortality was 9485 events. Compared to consuming 1-25 g alcohol/wk., the adjusted HR was 1.15 (95\% CI: 1.04-1.27) for 26-150 g/wk., 1.22 (95\% CI: 1.10-1.34) for $151-350 \mathrm{~g} /$ wk., 1.33 (95\% CI: 1.21-1.46) for $351-750 \mathrm{~g} / \mathrm{wk}$, and 1.57 (95\% CI: $1.30-1.90)$ for > $750 \mathrm{~g} / \mathrm{wk}$. Non-drinkers were associated with higher composite CVD, cancer, and mortality risk (adjusted $\mathrm{HR}=1.38 ; 95 \%$ CI: 1.29-1.49, Fig. 1). Consistently, the restricted cubic spline model resulted in a J-shape association with the lowest risk at $\sim 25 \mathrm{~g} / \mathrm{wk}$. of alcohol intake ( $\mathrm{p}$ for nonlinearity $<0.001$, Supplemental Figure 2a).

\section{Alcohol and CVD}

Light to moderate alcohol consumption (1-150 g/wk), but not heavy consumption $(>150 \mathrm{~g} / \mathrm{wk})$, was significantly associated with lower risk of CVD, relative to non-drinkers (Fig. 1). Alcohol consumption was inversely associated with myocardial infarction and heart failure in a dose-response manner $\left(P_{\text {trend }}<0.001\right.$, Fig. 2$)$. In contrast, there was a J-shape association between alcohol intake and stroke risk -- consumption of alcohol $1-150 \mathrm{~g} / \mathrm{wk}$. was significantly associated with lower stroke risk and $>350 \mathrm{~g} / \mathrm{wk}$. was associated with higher risk (Fig. 2). No significant association between alcohol intake and atrial fibrillation risk was observed (Fig. 2).

\section{Alcohol and cancer}

Individuals consuming $1-25 \mathrm{~g}$ alcohol/wk. had the lowest risk compared to other groups $(p<0.05$ for all, Fig. $1)$. Alcohol drinkers had marginally significant to significant higher risk of alcohol-attributed site-specific cancers (oral cavity, pharynx, larynx, esophageal, colorectum, anal, salivary gland, liver, and female breast) compared to non-drinkers $\left(P_{\text {trend }}=0.009\right.$, Fig. 2). In contrast, risk of non-alcohol-related cancers was significantly higher in non-drinkers, compared to individuals consuming 1-25 g/wk. alcohol (HR = 1.37; 95\% CI, 1.141.66, Fig. 2).

\section{Alcohol and mortality}

Non-drinkers and alcohol consumption $>25 \mathrm{~g} /$ wk. was associated with higher risk of all-cause mortality compared to 1-25 g/wk. (Fig. 1). Restricted cubic spline modeling resulted in a J-shape association (Supplemental Figure 2d). Similar patterns were observed for causespecific mortality (Fig. 2).

\section{Secondary analyses}

We found a significant interaction between alcohol and smoking ( $\mathrm{p}$ for interaction $<0.001$, Table 2 ), but not age, sex, and occupation, in relation to the composite CVD, cancer, and mortality risk. Due to small number of participants in $>750 \mathrm{~g} / \mathrm{wk}$. category, we combined this category with 351-750 g/wk. category in subgroup analyses. The association between alcohol intake and CVD, cancer, and mortality was stronger for never smokers and past smokers than current smokers (Table 2). For lung cancer specifically, individuals consuming 1-25 g alcohol/wk. had the lowest risk $(p<0.05$ compared to other groups).

The 2-year lag analyses and analyses after excluding former drinkers or very light drinkers $(1-4 \mathrm{~g} / \mathrm{wk})$ did not significantly change the result (Supplemental Table 24). Results of other interaction tests (age, sex, and occupation) and subgroup analyses are presented in Supplemental Tables 5-8. Analyses of different alcoholic beverage types and by cohort-specific tertiles of alcohol consumption are presented in Supplemental Tables 9 \& 10.

\section{Discussion}

In this large-scale prospective study including approximately 100,000 Chinese adults, we found that participants who reported consuming $1-150 \mathrm{~g} / \mathrm{wk}$. of alcohol 


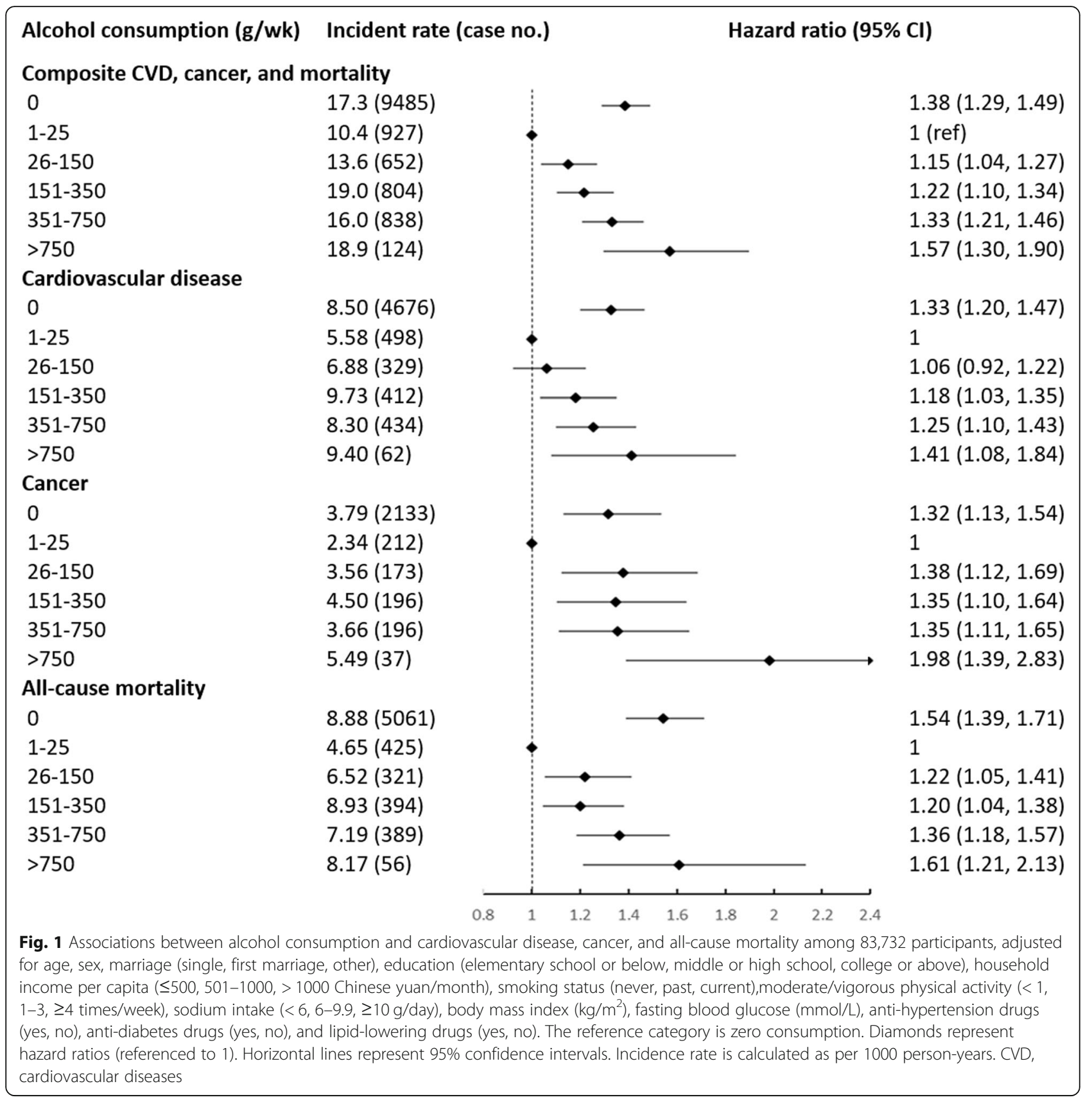

had lower risk for CVD, cancer, and mortality, relative to non-drinkers and heavy drinkers. This amount of alcohol intake is equivalent to approximately no more than 10 servings of alcohol/wk. The range identified is consistent with most global dietary guidelines for lowrisk drinking cutoffs (100 to $300 \mathrm{~g} / \mathrm{wk}$ ) [31]. However, in this Chinese cohort the lowest risk was observed among those with $\sim 25 \mathrm{~g} / \mathrm{wk}$, equivalent to $\sim 2$ servings per week. Because the mechanism for each CVD disorder or site-specific cancer is different, both individual and composite outcomes should be taken into consideration when developing dietary policy. Currently in China, there is no national policy on alcohol sales restrictions and no dietary guidelines for alcohol containing beverages. This study provides evidence for recommending light consumption of alcohol.

Our study in an Asian cohort supports prior observations of a J-shaped association between alcohol consumption and CVD [3, 17]. We found an overall lower CVD risk at consuming 1-150 g alcohol/wk. In secondary analyses, we identified different patterns for specific CVD. A J-shaped curve was found for stroke and an almost linear inverse association was found for myocardial infarction and heart failure. As previously suggested, 


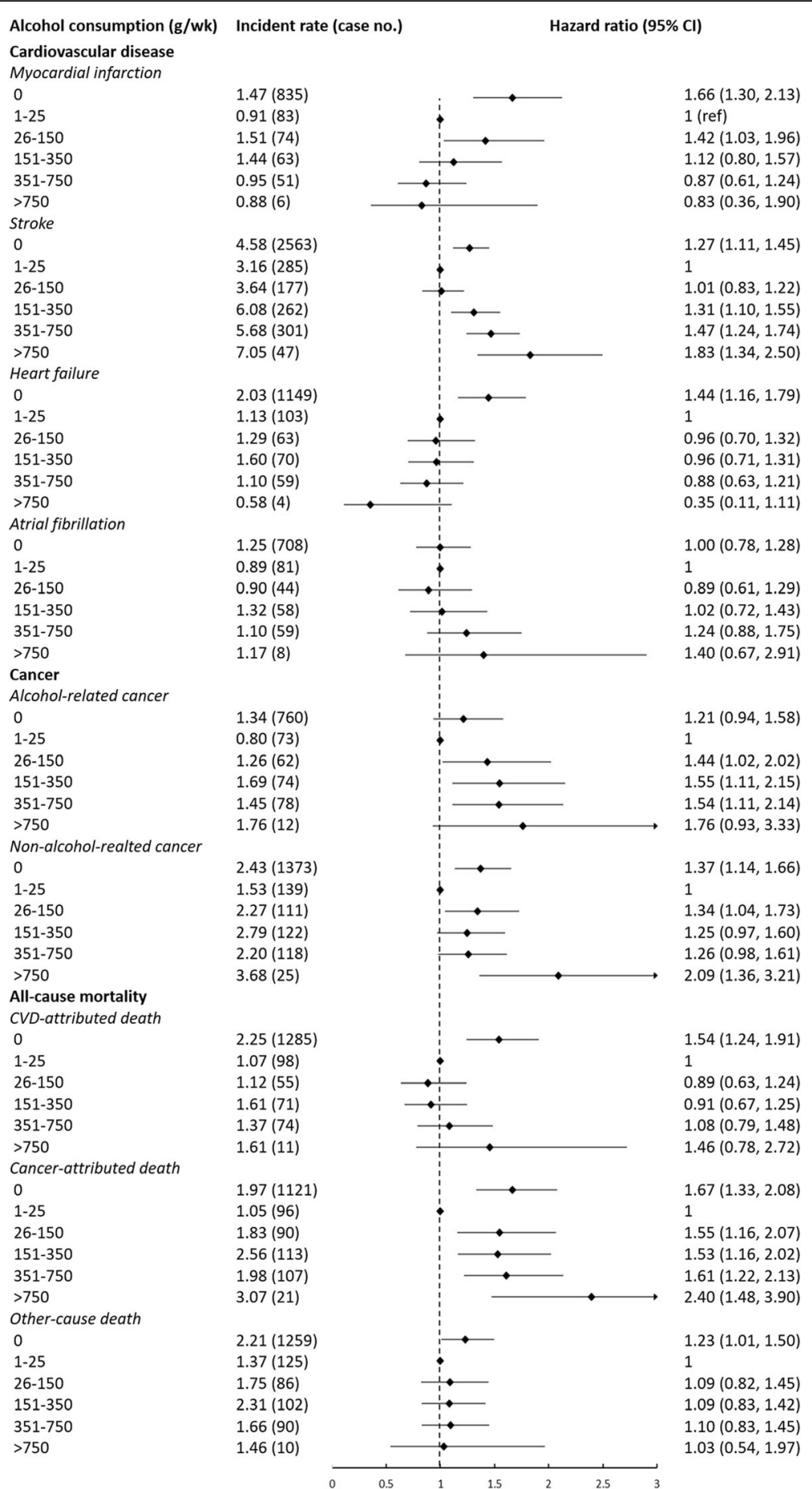

Fig. 2 Associations between alcohol consumption and specific chronic diseases and cause-specific mortality among 83,732 participants, adjusted for age, sex, marriage (single, first marriage, other), education (elementary school or below, middle or high school, college or above), household income per capita ( $\leq 500,501-1000,>1000$ Chinese yuan/month), smoking status (never, past, current), moderate/vigorous physical activity $(<1$, $1-3, \geq 4$ times/week), sodium intake $\left(<6,6-9.9, \geq 10 \mathrm{~g} /\right.$ day), body mass index $\left(\mathrm{kg} / \mathrm{m}^{2}\right)$, fasting blood glucose (mmol/L), anti-hypertension drugs (yes, no), anti-diabetes drugs (yes, no), and lipid-lowering drugs (yes, no). The reference category is zero consumption. Diamonds represent hazard ratios (referenced to 1). Horizontal lines represent 95\% confidence intervals. Incidence rate is calculated as per 1000 person-years. CVD, cardiovascular diseases 
Table 2 Association of alcohol consumption and CVD, cancer and mortality in different smoking status

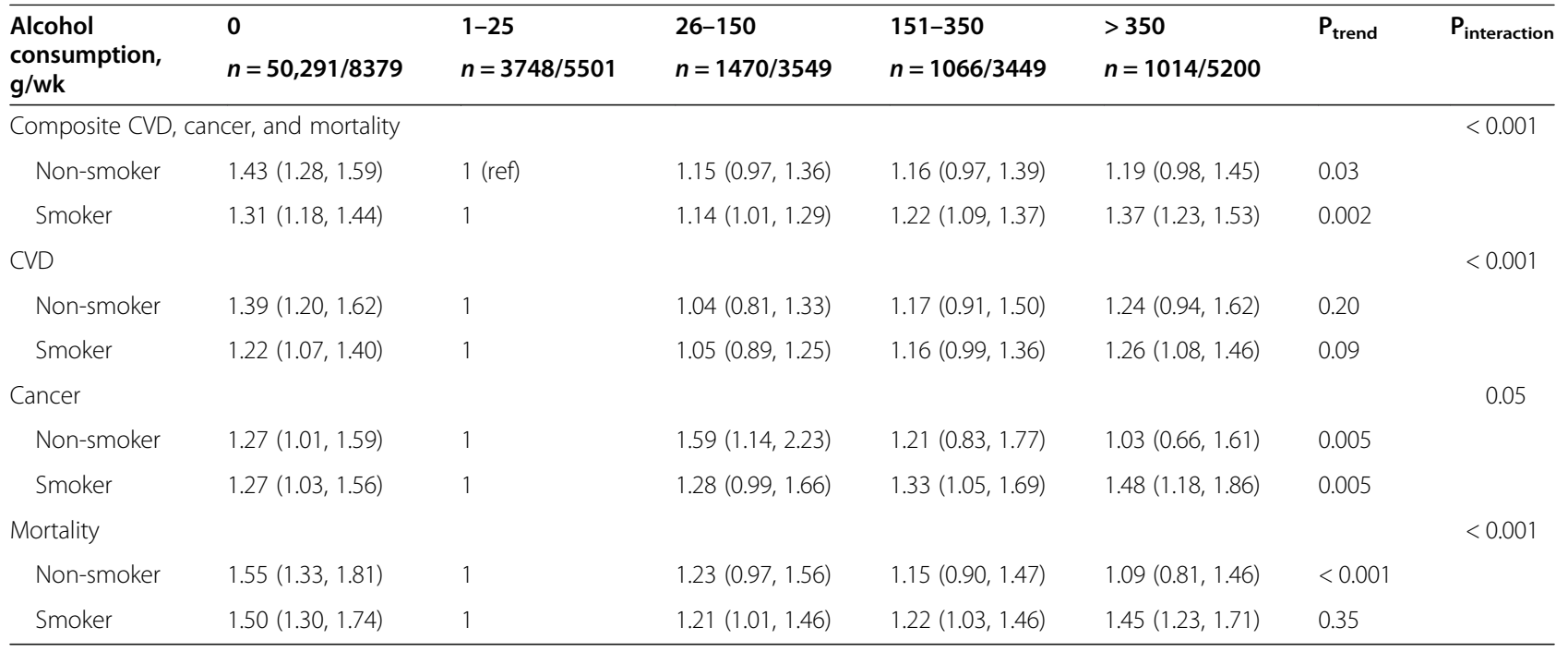

Values are hazard ratio and $95 \%$ confidence intervals. Numbers of non-smoker/smoker were listed for each category

Adjusted for age, sex, marriage (single, first marriage, other), education (elementary or below, middle or high school, college or above), household income per capita ( $\leq 500,501-1000,>1000$ Chinese yuan/month), moderate/vigorous physical activity $(<1,1-3, \geq 4$ times/week), sodium intake $(<6,6-9.9, \geq 10 \mathrm{~g} /$ day), body mass index $\left(\mathrm{kg} / \mathrm{m}^{2}\right)$, fasting blood glucose ( $\left.\mathrm{mmol} / \mathrm{L}\right)$, anti-hypertension drugs (yes, no), anti-diabetes drugs (yes, no), and lipid-lowering drugs (yes, no)

CVD cardiovascular diseases

alcohol could impact CVD risk via its favorable effects of raising HDL-C concentration and reducing inflammation, and its unfavorable effect of increasing blood pressure [6]. However, the impact of these risk factors on different CVDs (i.e., myocardial infarction vs stroke) could be different, thus potentially mediating their association with alcohol consumption. For example, the association of HDL-C and myocardial infarction could be stronger than its association with stroke [5, 32, 33]. Further, high blood pressure has consistently been identified as the strongest risk factor for stroke risk [32]. These factors may contribute to the different patterns in the associations between alcohol and myocardial infarction or heart failure, vs stroke.

We found J-shaped association between alcohol consumption and cancer, especially non-alcohol-related cancers. The causal pathway of alcohol is not fully understood for all cancer types. For female breast cancer, for example, alcohol could increase risk by altering levels of estrogen and estrogen receptors [34]. Hence, the inverse association between light alcohol intake $(\leq$ $25 \mathrm{~g} / \mathrm{wk}$ ) and overall cancer risk was likely driven by the lower risk of non-alcohol-related cancer. However, limited statistical power due to small number of individual non-alcohol-related cancer cases precluded us from addressing this hypothesis.

Our result showed that light to moderate alcohol intake was consistently associated with lower risk of total mortality and CVD- and cancer-specific mortality. The former observation is consistent with the result from a recent meta-analysis of 87 studies which found that compared to non-drinkers or high alcohol intakes, low- volume alcohol consumers (9.1-174 g alcohol/wk) had $14 \%$ lower risk of overall mortality [35].

In our Chinese cohort the association between alcohol consumption and major chronic disease risk was modified by smoking status. Because alcohol consumption and smoking are common addictive behaviors and covary, smoking could mask the effect of alcohol alone on health status [36-38]. As expected, although the protective association of light-to-moderate alcohol to CVD, cancer, and mortality was significant in both smokers and non-smokers, it was stronger in non-smokers. However, even for lung cancer, light alcohol consumption still showed lowest risk, compared to non-drinkers and heavy drinkers.

The potential biological mechanism of the overall beneficial effect of alcohol consumption is not fully understood, but may include altering cholesterol concentrations, especially HDL-C and triglyceride concentrations [22, 39], improving insulin sensitivity [39], decreasing the inflammatory process during cell signaling, decreasing platelet aggregation and blood clotting [40], and interaction with genetic variation in alcohol dehydrogenase polymorphism [41]. Although recent genetic epidemiological studies suggested that drinkers with ALDH2 deficiency had a more favorable profile for CVD risk factors, independent of alcohol consumption [6, 7], no relation was reported in non-drinkers.

This study was based on a large, community-based prospective Chinese cohort, with 16,044 incident events. Details on both exposures and outcomes were available, which enabled appropriate model adjustment and a relative comprehensive understanding of outcomes. The 
work is not without limitations. Potential confounders, such as diet quality, were not measured. To address this limitation, we adjusted for salt intake and other nutrition-related factors such as BMI, FBG, and lipid profiles to control of variation caused by residual confounding. In our previous cross-sectional analysis based on Kailuan Study 2014, salt intake was highly correlated with overall diet quality [21]. Alcohol consumption was calculated based on self-report frequency and usual amount of drinking. This could introduce measurement error, which might attenuate or magnify the association. However, drinking habits and amounts are common conversations with family and friends in many areas of China (e.g., Tangshan city) [42]. Therefore, self-reported consumption could be an acceptable measurement of the true intake with low risk of systematic bias, as supported by a significant correlation between self-report alcohol and longitudinal HDL-C concentration in the Kailuan Study. Of note, HDL-C concentration has been widely used for the validation study of self-report alcohol intake $[22,43]$. Binge drinking was not measured in this study. Genetic variation in ALDH2, and consequent metabolic response to alcohol (e.g., skin flash) were not available. The participants of the Kailuan Study lived in a traditional Chinese industrial community, which limits its generalization to other populations. However, the homogenous nature of the study population reduced variance in potential confounding and enhanced internal validity. Finally, due to the nature of Kailuan cohort, women were underrepresented (22.3\%), and most women (96.7\%) did not report consuming any alcoholic beverages, which is consistent with Chinese national nutritional survey and other reports [42]. This limited our capability to report sex-specific results as primary findings. Because women might be more vulnerable to alcohol due to pregnancy and risk of breast cancer than men [44], and data from a large scale prospective studies on Asian women are lacking on this topic.

\section{Conclusions}

Light to moderate alcohol consumption was associated with an overall beneficial outcome for CVD, cancer, and mortality compared to non-drinkers and heavy consumption. Individuals who consumed $\sim 25 \mathrm{~g}$ alcohol per wk. had the lowest risk of CVD, cancer, and mortality, relative to their peers.

\section{Supplementary Information}

The online version contains supplementary material available at https://doi. org/10.1186/s12937-021-00671-y.

Additional file 1 : Supplemental Table 1. Baseline comparison between excluded participants due to missing alcohol consumption data and included participants. Supplemental Table 2. Incidence rate and hazard ratios ( $95 \%$ confidence intervals) for CVD, cancer, and mortality by alcohol consumption at baseline. Supplemental Table 3. Hazard ratios (95\% confidence intervals) for CVD, cancer, and mortality by alcohol consumption, excluding former drinkers $(n=80,472)$. Supplemental Table 4. Hazard ratios (95\% confidence intervals) for CVD, cancer, and mortality by alcohol consumption, excluding participants with 1-4 $\mathrm{g}$ alcohol/wk $(n=80,320)$. Supplemental Table 5. Sex-specific association between alcohol consumption and CVD, cancer, and mortality. Supplemental Table 6. Association between alcohol consumption and CVD, cancer, and mortality subgroups by smoking status. Supplemental Table 7. Hazard ratios (95\% confidence intervals) for CVD, cancer, and mortality by alcohol consumption at baseline by age groups. Supplemental Table 8. Hazard ratios ( $95 \%$ confidence intervals) for CVD, cancer, and mortality by alcohol consumption at baseline by occupations. Supplemental Table 9. Hazard ratios (95\% confidence intervals) for CVD, cancer, and mortality associated with alcohol consumption at baseline by beverage types. Supplemental Table 10. Hazard ratios (95\% confidence intervals) for CVD, cancer, and mortality by alcohol consumption tertiles. Supplemental Figure 1. Flowchart of the study. Supplemental Figure 2. Restricted cubic spline model.

\section{Abbreviations}

Cl: Confidence interval; CVD: Cardiovascular disease; HDL-C: High-density lipoprotein cholesterol; HR: Hazard ratio; Hs-CRP: High sensitive C-reactive protein; ICD-10: International Classification of Diseases, 10th version; SES: Socio-economic status

\section{Acknowledgements}

None.

Authors' contributions

$X Z, Y L, X G$, and SW designed this research. $X Z$ and $Y L$ conducted research. SC, AX, and YW provided and monitored databases. $X Z$ and $Y L$ performed statistical analysis. XZ wrote paper. SL, AHL, MN, XG, SV, and SW made critical revision to the manuscript. XG and SW had primary responsibility for final content. All authors have read and approved the final manuscript.

\section{Funding}

This work was supported by the start-up grant from the College of Health and Human Development and the Department of Nutritional Sciences, the Pennsylvania State University.

Availability of data and materials

Data will be made available from the corresponding author upon reasonable request and approval.

Ethics approval and consent to participate

This study strictly followed the Declaration of Helsinki and was approved by the Ethics Review Board of the Kailuan General Hospital. Participants provided written informed consents.

\section{Consent for publication}

N/A.

\section{Competing interests}

The authors declare that they have no competing interests.

\section{Author details}

'Department of Nutritional Sciences, The Pennsylvania State University, 109 Chandlee Lab, University Park, PA 16802, USA. ${ }^{2}$ Department of Cardiology, Kailuan General Hospital, 57 Xinhua East Rd, Tangshan 063000, China. ${ }^{3}$ Slone Epidemiology Center, Boston University School of Medicine, Boston, USA. ${ }^{4}$ Jean Mayer USDA Human Nutrition Research Center on Aging, Tufts University, Boston, USA. ${ }^{5}$ Department of Family and Community Medicine and Public Health Sciences, The Pennsylvania State University College of Medicine, Hershey, USA. 
Received: 23 December 2020 Accepted: 27 January 2021

\section{Published online: 01 February 2021}

\section{References}

1. World Health Organization. Global information system on alcohol and health (GISAH). Switzerland: World Health Organization; 2016.

2. Smyth A, Teo KK, Rangarajan S, O'Donnell M, Zhang X, Rana P, et al. Alcohol consumption and cardiovascular disease, cancer, injury, admission to hospital, and mortality: a prospective cohort study. Lancet. 2015;386(10007): 1945-54.

3. Bell S, Daskalopoulou M, Rapsomaniki E, George J, Britton A, Bobak M, et al. Association between clinically recorded alcohol consumption and initial presentation of 12 cardiovascular diseases: population based cohort study using linked health records. BMJ. 2017;356:j909.

4. Ricci C, Wood A, Muller D, Gunter MJ, Agudo A, Boeing H, et al. Alcohol intake in relation to non-fatal and fatal coronary heart disease and stroke: EPIC-CVD case-cohort study. BMJ. 2018;361:k934.

5. Wood AM, Kaptoge S, Butterworth AS, Willeit P, Warnakula S, Bolton T, et al. Risk thresholds for alcohol consumption: combined analysis of individualparticipant data for 599912 current drinkers in 83 prospective studies. Lancet. 2018;391(10129):1513-23.

6. Millwood IY, Walters RG, Mei XW, Guo Y, Yang L, Bian Z, et al. Conventional and genetic evidence on alcohol and vascular disease aetiology: a prospective study of 500000 men and women in China. Lancet. 2019; 393(10183):1831-42.

7. Holmes MV, Dale CE, Zuccolo L, Silverwood RJ, Guo Y, Ye Z, et al. Association between alcohol and cardiovascular disease: Mendelian randomisation analysis based on individual participant data. BMJ. 2014;349:94164.

8. IARC Working Group on the Evaluation of Carcinogenic Risks to Humans. Alcohol consumption and ethyl carbamate. France: IARC press, International Agency for Research on Cancer; 2010.

9. Bagnardi V, Rota M, Botteri E, Tramacere I, Islami F, Fedirko V, et al. Alcohol consumption and site-specific cancer risk: a comprehensive dose-response meta-analysis. Br J Cancer. 2014;112(3):580-93.

10. Rehm J, Room R, Graham K, Monteiro M, Gmel G, CTJA S. The relationship of average volume of alcohol consumption and patterns of drinking to burden of disease: an overview. Addiction. 2003;98(9):1209-28.

11. Xi B, Veeranki SP, Zhao M, Ma C, Yan Y, Mi J. Relationship of alcohol consumption to all-cause, cardiovascular, and cancer-related mortality in U. S. adults. J Am Coll Cardiol. 2017;70(8):913-22.

12. Goedde HW, Agarwal DP, Harada S, Meier-Tackmann D, Ruofu D, Bienzle U, et al. Population genetic studies on aldehyde dehydrogenase isozyme deficiency and alcohol sensitivity. Am J Hum Genet. 1983;35(4):769-72.

13. Bray F, Ferlay J, Soerjomataram I, Siegel RL, Torre LA, Jemal A. Global cancer statistics 2018: GLOBOCAN estimates of incidence and mortality worldwide for 36 cancers in 185 countries. CA Cancer J Clin. 2018;68(6):394-424.

14. Shirakawa T, Yamagishi K, Yatsuya H, Tanabe N, Tamakoshi A, Iso H, et al. Alcohol consumption and mortality from aortic disease among Japanese men: the Japan collaborative cohort study. Atherosclerosis. 2017;266:64-8.

15. Nelson DE, Jarman DW, Rehm J, Greenfield TK, Rey G, Kerr WC, et al. Alcohol-attributable cancer deaths and years of potential life lost in the United States. Am J Public Health. 2013;103(4):641-8.

16. Griswold MG, Fullman N, Hawley C, Arian N, Zimsen SRM, Tymeson HD, et al. Alcohol use and burden for 195 countries and territories, 1990-2016: a systematic analysis for the Global Burden of Disease Study 2016. Lancet. 2018;392(10152):1015-35.

17. Larsson SC, Wallin A, Wolk A, Markus HS. Differing association of alcohol consumption with different stroke types: a systematic review and metaanalysis. BMC Med. 2016;14(1):178.

18. Zhang C, Qin $Y Y, C$ hen $Q$, Jiang $H$, Chen $X Z$, Xu CL, et al. Alcohol intake and risk of stroke: a dose-response meta-analysis of prospective studies. Int J Cardiol. 2014;174(3):669-77.

19. McCullough ML, Feskanich D, Rimm EB, Giovannucci EL, Ascherio A, Variyam $\mathrm{JN}$, et al. Adherence to the dietary guidelines for Americans and risk of major chronic disease in men. Am J Clin Nutr. 2000;72(5):1223-31.

20. McCullough ML, Feskanich D, Stampfer MJ, Rosner BA, Hu FB, Hunter DJ, et al. Adherence to the dietary guidelines for Americans and risk of major chronic disease in women. Am J Clin Nutr. 2000;72(5):1214-22.

21. Wu S, An S, Li W, Lichtenstein AH, Gao J, Kris-Etherton PM, et al. Association of trajectory of cardiovascular health score and incident cardiovascular disease. JAMA Netw Open. 2019;2(5):e194758.
22. Huang S, Li J, Shearer GC, Lichtenstein AH, Zheng X, Wu Y, et al. Longitudinal study of alcohol consumption and HDL concentrations: a community-based study. Am J Clin Nutr. 2017;105(4):905-12.

23. Cao Y, Willett WC, Rimm EB, Stampfer MJ, Giovannucci EL. Light to moderate intake of alcohol, drinking patterns, and risk of cancer: results from two prospective US cohort studies. BMJ. 2015;351:h4238.

24. Jin C, Chen S, Vaidya A, Wu Y, Wu Z, Hu FB, et al. Longitudinal change in fasting blood glucose and myocardial infarction risk in a population without diabetes. Diabetes Care. 2017:40(11):1565-72.

25. Li Y, Huang Z, Jin C, Xing A, Liu Y, Huangfu C, et al. Longitudinal change of perceived salt intake and stroke risk in a Chinese population. Stroke. 2018; 49(6):1332-9.

26. Ho KKL, Pinsky JL, Kannel WB, Levy D. The epidemiology of heart failure: the Framingham Study. J Am Coll Cardiol. 1993;22(4):A6-A13.

27. Li S, Cheng J, Cui L, Gurol ME, Bhatt DL, Fonarow GC, et al. Cohort study of repeated measurements of serum urate and risk of incident atrial fibrillation. J Am Heart Assoc. 2019;8(13):e012020.

28. Feng X, Wang G, Lyu Z, Chen S, Wei L, Li X, et al. The association between fasting blood glucose trajectory and cancer risk in Chinese population without diabetes. Int J Cancer. 2020;147(4):958-66.

29. Wu Z, Jin C, Vaidya A, Jin W, Huang Z, Wu S, et al. Longitudinal patterns of blood pressure, incident cardiovascular events, and all-cause mortality in normotensive diabetic people. Hypertension. 2016;68(1):71-7.

30. Wu Z, Huang Z, Jin W, Rimm EB, Lichtenstein AH, Kris-Etherton PM, et al. Peripheral inflammatory biomarkers for myocardial infarction Risk: a prospective community-based study. Clin Chem. 2017;63(3):663-72.

31. Kalinowski A, Humphreys K. Governmental standard drink definitions and low-risk alcohol consumption guidelines in 37 countries. Addiction. 2016;111(7):1293-8.

32. Woodward M, Barzi F, Feigin V, Gu D, Huxley R, Nakamura K, et al. Associations between high-density lipoprotein cholesterol and both stroke and coronary heart disease in the Asia Pacific region. Eur Heart J. 2007;28(21):2653-60.

33. Emerging Risk Factors C, Di Angelantonio E, Sarwar N, Perry P, Kaptoge S, Ray KK, et al. Major lipids, apolipoproteins, and risk of vascular disease. JAMA. 2009;302(18):1993-2000.

34. Suzuki R, Orsini N, Mignone L, Saji S, Wolk A. Alcohol intake and risk of breast cancer defined by estrogen and progesterone receptor status--a meta-analysis of epidemiological studies. Int J Cancer. 2008;122(8):1832-41.

35. Stockwell T, Zhao J, Panwar S, Roemer A, Naimi T, Chikritzhs T. Do "moderate" drinkers have reduced mortality risk? A systematic review and meta-analysis of alcohol consumption and all-cause mortality. J Stud Alcohol Drugs. 2016;77(2):185-98.

36. Blot WJ, McLaughlin JK, Winn DM, Austin DF, Greenberg RS, Preston-Martin $\mathrm{S}$, et al. Smoking and drinking in relation to oral and pharyngeal cancer. Cancer Res. 1988;48(11):3282-7.

37. Petti S, Masood M, Scully C. The magnitude of tobacco smoking-betel quid chewing-alcohol drinking interaction effect on oral cancer in South-East Asia. A meta-analysis of observational studies. PLoS One. 2013;8(11):e78999.

38. Xu WH, Zhang XL, Gao YT, Xiang YB, Gao LF, Zheng W, et al. Joint effect of cigarette smoking and alcohol consumption on mortality. Prev Med. 2007:45(4):313-9.

39. Davies MJ, Baer DJ, Judd JT, Brown ED, Campbell WS, Taylor PR. Effects of moderate alcohol intake on fasting insulin and glucose concentrations and insulin sensitivity in postmenopausal women: a randomized controlled trial. JAMA. 2002;287(19):2559-62.

40. Zakhari S. Alcohol and the cardiovascular system: molecular mechanisms for beneficial and harmful action. Alcohol Health Res World. 1997;21(1):21-9.

41. Hines LM, Stampfer MJ, Ma J, Gaziano JM, Ridker PM, Hankinson SE, et al. Genetic variation in alcohol dehydrogenase and the beneficial effect of moderate alcohol consumption on myocardial infarction. N Engl J Med. 2001:344(8):549-55.

42. Cochrane J. Alcohol use in China. Alcohol Alcohol. 2003;38(6):537-42.

43. Glovannucci E, Colditz G, Stampfer MJ, Rimm EB, Litin L, Sampson L, et al. The assessment of alcohol consumption by a simple self-administered questionnaire. Am J Epidemiol. 1991;133(8):810-7.

44. Zheng YL, Lian F, Shi Q, Zhang C, Chen YW, Zhou YH, et al. Alcohol intake and associated risk of major cardiovascular outcomes in women compared with men: a systematic review and meta-analysis of prospective observational studies. BMC Public Health. 2015;15(1):773.

\section{Publisher's Note}

Springer Nature remains neutral with regard to jurisdictional claims in published maps and institutional affiliations. 